\title{
Arbitrarily Accurate Dynamical Control in Open Quantum Systems
}

\author{
Kaveh Khodjasteh, ${ }^{1}$ Daniel A. Lidar, ${ }^{2}$ and Lorenza Viola ${ }^{1}$ \\ ${ }^{1}$ Department of Physics and Astronomy, Dartmouth College, 6127 Wilder Laboratory, Hanover, NH 03755, USA \\ ${ }^{2}$ Departments of Chemistry, Electrical Engineering, and Physics, and \\ Center for Quantum Information Science 85 Technology, University of Southern California, Los Angeles, CA 90089, USA
}

\begin{abstract}
We show that open-loop dynamical control techniques may be used to synthesize unitary transformations in open quantum systems in such a way that decoherence is perturbatively compensated for to a desired (in principle arbitrarily high) level of accuracy, which depends only on the strength of the relevant errors and the achievable rate of control modulation. Our constructive and fully analytical solution employs concatenated dynamically corrected gates, and is applicable independently of detailed knowledge of the system-environment interactions and environment dynamics. Explicit implications for boosting quantum gate fidelities in realistic scenarios are addressed.

PACS numbers: 03.67.Pp, 03.67.Lx, 03.65Yz, 07.05.Dz
\end{abstract}

The demand for an exquisite degree of control over the dynamics of open quantum systems is widespread across quantum physics and engineering, ranging from high-resolution spectroscopy and chemical reaction control [1], to quantum-limited metrology [2] and quantum information processing (QIP) [3]. Achieving a sufficiently small 'error per gate' (EPG) is, in particular, an essential ingredient to ensuring that fault-tolerant quantum computation is possible in spite of the decoherence that inevitably plagues real-world devices. While closed-loop techniques, in the form of fault-tolerant quantum error correction (QEC) [4, 5], offer thus far the only complete prescription to meet this challenge, open-loop dynamical $Q E C$ is emerging as a promising alternative. Inspired by coherent averaging in magnetic resonance [6] and exemplified in its simplest form by dynamical decoupling (DD) [7-9], dynamical QEC aims to suppress the interaction between the system and its quantitatively unspecified environment through suitable sequences of unitary operations. Recently, DD has enabled decoherence-protected storage in QIP platforms as diverse as electron-nuclear systems [10], photonics qubits [11], and trapped ions [12], as well as found application in suppressing collisional decoherence in cold atoms [13].

As the gap between theory and implementations shrinks, and a growing experimental effort is devoted to robust manipulation of quantum states, it is imperative that realistic constraints be accommodated from the outset in dynamical QEC design. In practice, control resources always entail finite power and bandwidth, thus precluding instantaneous ('bang-bang' [7]) pulses. A path toward decoherence-protected unitary operations was recently proposed based on dynamically corrected gates (DCGs) [14]. A DCG may be viewed as a composite quantum gate constructed from individual ("primitive') building blocks whose errors combine non-linearly to achieve a substantially smaller net error. If $\tau_{\min }$ is the minimum duration over which each primitive gate is effected ('switching time') and $\left\|H_{e}\right\|$ the strength of the error-inducing Hamiltonian, DCGs remove the effect of
$H_{e}$ to the leading (first) order, that is, the resulting EPG scales as $\mathcal{O}\left[\left(\tau_{\min }\left\|H_{e}\right\|\right)^{2}\right]$. This prompts the following key question: Can one make DCGs as accurate as desired, using realistic control resources? The answer is not obvious. Schemes capable of arbitrarily suppressing decoherence during storage have been identified - notably, concatenated DD [8] and recent optimized protocols $[9,15]-$ but, thus far, only in the bang-bang limit. With bounded controls, decoherence suppression up to the second order (with leading corrections $\left.\mathcal{O}\left[\left(\tau_{\min }\left\|H_{e}\right\|\right)^{3}\right]\right)$ may be achieved by using a time-symmetrized Euler DD (EDD) protocol [16] (see also [17]) - however, this procedure extends neither to generic quantum gates nor to generic open quantum systems $[14,18]$.

Here we show that decoherence suppression can in principle be pushed to an order limited only by the strength of the relevant errors and the achievable rate of control modulation. We do this by combining DCG constructions with recursive design - resulting in concatenated DCGs. While perturbative in nature, our solution is fully analytical, laying the foundation for rigorous complexity analysis and optimization in dynamical QEC. Not only do concatenated DCGs exist for arbitrary finite-dimensional open quantum systems with a bounded $\left\|H_{e}\right\|$, but they are also highly portable, in the sense that no quantitative knowledge of the underlying interaction Hamiltonian is assumed, beyond its algebraic form. Since arbitrarily accurate open-loop compensation techniques for classical (static) control errors are known [19], our results imply that no fundamental limitation arises due to a quantum (dynamic) bath. From a practical standpoint, concatenated DCGs offer the first systematic feedback-free framework for designing quantum gates which can achieve the arbitrarily high levels of protection against decoherence demanded by high-fidelity quantum control, and in particular QIP.

Control-theoretic setting. - Let $S$ be the target quantum system, coupled to its quantum bath $B$ via an interaction Hamiltonian $H_{S B}$, with respective Hilbert space $\mathcal{H}_{S}$ and $\mathcal{H}_{B}$. We assume that the total error Hamilto- 
nian $H_{e}$ may be described by a joint time-independent operator of the following form:

$$
H_{e}=H_{S, e}+H_{S B}+H_{B} \equiv \sum_{\alpha} S_{\alpha} \otimes B_{\alpha},
$$

where the contribution $H_{S, e}$ accounts for undesired 'drift' terms possibly present in the system's internal Hamiltonian, $\left\{S_{\alpha}\right\}$ is a Hermitian basis of operators acting on $\mathcal{H}_{S}$, and $B_{\alpha}$ are bounded (potentially unknown) operators acting on $\mathcal{H}_{B}$. The vector space $\Omega_{e}$ spanned by $\left\{S_{\alpha} \otimes B_{\alpha}\right\}$, with non-zero $B_{\alpha}$, defines the error model and is uniquely determined by the system components $\left\{S_{\alpha}\right\}$ appearing in the expansion of $H_{e}$ in Eq. (1). In dynamical QEC, a classical controller is adjoined to $S$ through a time-dependent Hamiltonian $H_{\text {ctrl }}(t)$. Several constraints may restrict, in reality, the degree of control that is available through $H_{\mathrm{ctrl}}(t)$. In particular, we account for realistic control modulations to be bounded in amplitude and spectral bandwidth by requiring that $\left\|H_{\text {ctrl }}(t)\right\|<\infty$ and $\tau_{\min }>0$, respectively. In an ideal situation where $H_{S B}=0=H_{S, e}$, the errors arise from imperfections in the controller only. Our goal here is to present a proof-of-concept for robustness against decoherence errors during gates, thus we ignore such contributions henceforth. We furthermore assume that universal control over $S$ is achievable [3] (see [14] for departures from this 'minimal' setting).

Consider a unitary operation (gate) $Q$ on $S$, which is ideally realized by letting $H_{\text {ctrl }}(t)=H_{Q}(t)$ over an interval $\left[t_{1}, t_{1}+\tau\right]$. We use $Q[\tau]$ or $Q$ to denote this implementation of $Q$ when there is no ambiguity. If $H_{e} \neq 0$, the joint propagator is $U_{Q[\tau]}=\mathcal{T} \exp \left[-i \int_{t_{1}}^{t_{1}+\tau}\left(H_{Q}(t)+\right.\right.$ $\left.\left.H_{e}\right) d t\right]$ ( $\hbar=1$ and $\mathcal{T}$ denotes time-ordering). The effect of $H_{e}$ may be isolated through a Hermitian 'error action operator' $E_{Q[\tau]}$ [14], where $U_{Q[\tau]}=Q \exp \left(-i E_{Q[\tau]}\right)$. Let the initial states of $S$ and $B$ be $\rho_{S}^{0}=|\psi\rangle\langle\psi|$ and $\rho_{B}$. Ideally, the action of $Q$ would result in $\rho_{S}^{0}(\tau)=Q \rho_{S}^{0} Q^{\dagger}$, while $B$ evolves independently. In contrast, the actual evolution is coupled and $\rho_{S}(\tau)=\operatorname{Tr}_{B}\left(U_{Q[\tau]} \rho_{S}^{0} \otimes\right.$ $\left.\rho_{B} U_{Q[\tau]}^{\dagger}\right)$. An appropriate performance measure for control is the trace-norm distance $\Delta \equiv\left\|\rho_{S}(\tau)-\rho_{S}^{0}(\tau)\right\|_{1}[3]$. One can show that $\Delta \leq\left\|\bmod _{B}\left(E_{Q[\tau]}\right)\right\|$, independently of the initial state, where $\bmod _{B}(E) \equiv E-\frac{1}{\operatorname{Tr}\left(I_{S}\right)} I_{S} \otimes \operatorname{Tr}_{S}(E)$ is a projector that removes the pure-bath terms in $E$ $[20,21]$. Thus, $\eta \equiv\left\|\bmod _{B}\left(E_{Q[\tau]}\right)\right\|$ may be taken to quantify the resulting EPG.

Given a desired unitary gate $Q$, our task is to synthesize a control modulation that approximates $Q$ with an EPG scaling as $\mathcal{O}\left(\tau_{\min }^{\ell+1}\right)$, for an arbitrary positive integer $\ell$ and compatible with the stated control constraints. Any such construction defines an $\ell$-th order DCG, DCG ${ }^{[\ell]}$. "Naïve" implementations that simply correspond to turning on, say, a constant $H_{\mathrm{ctrl}}(t)$ for a duration $\tau$ yield an EPG that scales (approximately) linearly with $\tau$ in the presence of $H_{e}$ and are included as $\ell=0$.
Primitive gates are correspondingly denoted by $\left\{Q^{[0]}\right\}$. Free evolution under $H_{e}\left[H_{\mathrm{ctrl}}(t) \equiv 0\right]$ may be viewed also as a zeroth order 'no-operation' (NOOP) gate. Consider a combined gate $\left(Q_{1} \cdots Q_{N}\right)\left[\sum \tau_{i}\right]$ formed as a sequence of $N$ primitive gates $Q_{N}^{[0]}\left[\tau_{N}\right] \cdots Q_{2}^{[0]}\left[\tau_{2}\right] Q_{1}^{[0]}\left[\tau_{1}\right]$ applied back to back. We may compute the total error for the combined gate using

$$
\begin{aligned}
& E_{\left(Q_{1} \cdots Q_{N}\right)\left[\sum \tau_{i}\right]}=E_{Q_{1}\left[\tau_{1}\right]}+ \\
& +P_{1}^{\dagger} E_{Q_{2}\left[\tau_{2}\right]} P_{1}+\cdots+P_{N-1}^{\dagger} E_{Q_{N}\left[\tau_{N}\right]} P_{N-1}+E_{2+},
\end{aligned}
$$

where $P_{j}=Q_{j} \cdots Q_{1}, j=1, \ldots, N-1$, is the 'partial' control propagator at the end of the $j$ th segment [22] and $\left\|E_{2+}\right\|=\mathcal{O}\left(\sum_{i, j} \|\left[E_{Q_{i}\left[\tau_{i}\right]}, E_{Q_{j}\left[\tau_{j}\right]} \|\right)\right.$ includes the leading second-order corrections. Note that individual EPGs do not simply add up, but are 'modulated' by the control trajectory: in dynamical QEC, this provides the basic mechanism enabling the effect of $H_{e}$ to be perturbatively canceled, without quantitative knowledge of the bath.

Concatenated DCGs: Construction and performance bound.- Our strategy to achieve an arbitrary order of cancellation is to invoke a recursive construction, that generates $(\ell+1)^{\text {th }}$-order gates using $\ell^{\text {th }}$-order building blocks, close in spirit to concatenated DD [8]. In what follows, given a target gate $Q$, we shall term an $\ell$-th order balance pair as any pair of gates $\left(I_{Q}^{[\ell]}, Q_{*}^{[\ell]}\right)$ whose errors coincide up to the leading $(\ell+1)^{\mathrm{th}}$-order, that is, $\bmod _{B}\left(E_{I_{Q}}\right)=\bmod _{B}\left(E_{Q_{*}}\right)+\mathcal{O}\left(\tau_{\min }^{\ell+2}\right)$.

The first step in the recursion requires demonstrating that, given $Q$, a corresponding $\mathrm{DCG}^{[1]}$ can be constructed out of the available universal set of primitive gates. As established in [14], this necessitates two main ingredients: (i) An EDD protocol for generating a $\mathrm{DCG}^{[1]}$-implementation of NOOP; (ii) A zeroth-order balance pair $\left(I_{Q}^{[0]}, Q_{*}^{[0]}\right)$, with the same leading order error $E_{Q_{*}}$ belonging to a 'correctable error space' $\Omega_{\mathcal{D}} \supseteq \Omega_{e}$. In order to achieve (i), a set of unitary gates $\mathcal{D}=\left\{D_{i}\right\}_{i=1}^{d}$ is identified, such that the map $\Pi_{\mathcal{D}}[E] \equiv \frac{1}{d} \sum_{i=1}^{d} D_{i}^{\dagger} E D_{i}$ 'decouples' all errors $E$ in $\Omega_{\mathcal{D}}: \bmod _{B}\left(\Pi_{\mathcal{D}}[E]\right)=0$ [7]. In EDD [16], $\mathcal{D}$ represents a group $\mathcal{G}$ (faithfully and projectively), with order $|\mathcal{G}|=d$ and a set of $m_{\ell}$ generators $\Gamma=\left\{F_{j}\right\}$. Let $G(\mathcal{G}, \Gamma)$ denote the Cayley graph of $\mathcal{G}$ with respect to $\Gamma$. The required EDD sequence (of length $N_{1}=d m$ ) is constructed by consecutively applying the generators $F_{j}$ as gates, in the order determined by an Eulerian cycle on $G(\mathcal{G}, \Gamma)$, starting (and ending) at the identity vertex, $I_{S}$. Suppose that each $F_{j}$ is implemented as a zeroth order gate with $E_{F_{j}} \in \Omega_{\mathcal{D}}$. Then, using Eq. (2) and the decoupling property of $\mathcal{G}$, the net error $\eta_{\mathrm{EDD}}=\mathcal{O}\left(\max _{j}\left\|E_{F_{j}}\right\|^{2}\right)=\mathcal{O}\left(\tau_{\text {min }}^{2}\right)$, as desired.

In order to extend the EDD construction to a gate $Q$ other than NOOP, some information about how primitive gates are implemented is required, due to a NoGo theorem for 'control-oblivious' design proved in [14]; balance pairs are required as ingredient (ii) precisely for this pur- 
pose. Once such a balance pair $\left(I_{Q}^{[0]}, Q_{*}^{[0]}\right)$ is found, a $\mathrm{DCG}^{[1]}$ sequence for $Q$ is obtained by 'augmenting' the Cayley graph for NOOP: $I_{Q}^{[0]}$ gates are inserted in the EDD sequence at points where the corresponding Eulerian path visits each non-identity vertex for the first time, and finally $Q_{*}^{[0]}$ is applied after the last $I_{S}$ vertex. The resulting error for the combined sequence is then given by $\left\|\bmod _{B}\left(E_{Q^{[1]}}\right)\right\|=\left\|\bmod _{B}\left(E_{\mathrm{EDD}}+\Pi_{\mathcal{D}}\left(E_{Q_{*}}\right)\right)\right\|=0$ plus corrections given by error $\eta_{\mathrm{DCG}}^{[1]}=\mathcal{O}\left(\tau_{\min }^{2}\right)$, as desired.

Our balance pair construction "strikes a balance" by stretching control profiles. Not only does this ensure a fully portable recipe in terms of control inputs (note that previous DCG constructions [14], required access to signreversed control profiles), but it is in fact crucial for producing balance pairs of high-order gates. In practice, a stretched control input results in slower gates. More concretely, for a gate stretched by a factor $r$, the gating Hamiltonian is "stretched" by a factor $r: H_{Q}(t) \mapsto$ $H_{Q}\left[t_{1}+r\left(t-t_{1}\right)\right] / r$; thus the same target gate $Q$ is approximated, but with a different EPG. The stretched gate is symbolically indicated by $Q[r \tau]$ but all gate constructions are still subject to the original bandwidth/power constraints. The general recipe for balance pairs of any order $\ell$ is given in terms of $Q^{[\ell]}$ and $Q^{-1,[\ell]}\left(\ell^{\text {th }}\right.$ order implementations of $Q$ and its inverse) by the following pair of composite gates [21]:

$$
\begin{aligned}
I_{Q}^{[\ell]} & =Q^{-1,[\ell]}[\tau] Q^{[\ell]}\left[2^{1 /(\ell+1)} \tau\right], \\
Q_{*}^{[\ell]}[\tau] & =Q^{[\ell]}[\tau] Q^{-1,[\ell]}[\tau] Q^{[\ell]}[\tau] .
\end{aligned}
$$

Note that $I_{Q}^{[\ell]}$ implements the NOOP gate $\left(I_{S}\right)$ over a duration of $\tau+2^{1 /(\ell+1)} \tau$, while $Q_{*}^{[\ell]}$ implements $Q$ over $3 \tau$. Setting $\ell=0$ thus completes the construction of $1^{\text {st }}$-order gates using $0^{\text {th }}$-order building blocks.

A (universal) set of gates $\left\{Q^{[\ell]}\left[\tau_{\ell}\right]\right\}$ with $\mathrm{EPG}=$ $\mathcal{O}\left(\tau_{\min }^{\ell+1}\right)$ can be constructed recursively for arbitrary $\ell \geq 1$ at this point. Let $\Omega_{e}^{[\ell]}$ denote the error model for all $\ell^{\text {th }}$-order gates: $E_{Q^{[\ell]}} \in \Omega_{e}^{[\ell]}[23]$. We can then identify the smallest DD group $\mathcal{G}^{[\ell]}$ that decouples $\Omega_{e}^{[\ell]}$, with $m_{\ell}$ group generators $\Gamma^{[\ell]}=\left\{F_{j}^{[\ell]}\right\}$, and a corresponding Cayley graph $G\left(\mathcal{G}^{[\ell]}, \Gamma^{[\ell]}\right)$. We modify this graph by attaching self-directed edges representing $I_{Q}^{[\ell]}$ to all vertexes except $I_{S}$, and add a new vertex $Q$ by connecting it to the $I_{S}$-vertex through an edge representing $Q_{*}^{[\ell]}$. By construction, every edge in this graph represents a $\mathrm{DCG}^{[\ell]}$. We now proceed as in first-order DCGs, and implement the sequence $Q^{[\ell+1]}\left[\tau_{\ell+1}\right]$ by following the $N_{\ell}=d_{\ell} m_{\ell}+d_{\ell}$ edges of an Eulerian path on the modified graph for $G\left(\mathcal{G}^{[\ell]}, \Gamma^{[\ell]}\right)$, starting at $I_{S}$ and stopping at $Q$, and applying the corresponding $Q_{i}^{[\ell]}$ gates back to back. If the latter are implemented with duration $\tau_{\ell}$ (before stretching), then the combined total duration $\tau_{\ell+1}$ satisfies:

$$
\tau_{\ell+1}=\left[d_{\ell} m_{\ell}+\left(d_{\ell}-1\right)\left(1+2^{1 /(\ell+1)}\right)+3\right] \tau_{\ell} .
$$

By iterating, we obtain a cascade of stretched primitive control profiles of duration $\tau_{\ell} \lesssim\left(\chi_{\ell}\right)^{\ell} \tau_{0}$, where $\chi_{\ell}=$ $d_{\ell}\left(m_{\ell}+3\right)$ [24]. Starting with the primitive gates $[\ell=0]$ of duration $\tau_{0}$, the above construction generates a DCG ${ }^{[\ell]}$ for any $Q$ and $\ell$, with a net error upper bounded by:

$$
\eta_{\mathrm{DCG}}^{[\ell]}<c\left(\chi_{\ell}\right)^{\ell^{2}} \tau_{0}\left\|H_{S B}+H_{S, e}\right\|\left(4 \chi_{\ell} \tau_{0}\left\|H_{e}\right\|\right)^{\ell},
$$

where $c=\mathcal{O}(1)$. While a detailed proof will be presented elsewhere [21], two main steps are involved. First, we prove that for any gate $Q^{[\ell]}[\tau]$, owing to the recursive design of the sequence, the Magnus expansion of the error $E_{Q^{[\ell]}}$ in terms of the toggling frame error Hamiltonian $H_{e}(t)=U_{\text {ctrl }}^{\dagger}(t) H_{e} U_{\text {ctrl }}(t)$ contains only terms that start at $\mathcal{O}\left(\tau^{\ell+1}\right)$ (modulo the pure bath terms). Second, we bound these higher order terms (hence $\| \bmod _{B}\left(E_{Q^{[\ell]}} \|\right)$ ) using standard operator inequalities.

Concatenated DCGs: Analysis and applications.From a practical perspective, our result above shows that concatenated DCGs offer concrete error reduction over primitive gates and $\mathrm{DCG}^{[1]}$ : for any fixed achievable switching time $\tau_{0}$, minimizing the bound in Eq. (4) yields an optimal concatenation level $\ell_{\mathrm{opt}}=$ $\left\lfloor-\frac{1}{2}\left(\log _{\chi}\left(4\left\|H_{e}\right\| \tau_{0}\right)+1\right)\right\rfloor$, where $\lfloor x\rfloor$ is the largest integer $\leq x$, and $\chi \equiv \chi_{\ell_{\mathrm{opt}}}$. Substituting $\ell_{\mathrm{opt}}$ into Eq. (4) yields an EPG bound that may enable scalable QIP even if primitive gates, whose EPG is given by $\left\|H_{S B}+H_{S, e}\right\| \tau_{0}$, are above the accuracy threshold of (non-Markovian) fault-tolerant QEC [25]. Ultimately, the viability of a concatenated DCG will be dictated by system-dependent implementation trade-offs, based on both the total gate duration and minimum switching time.

To illustrate our general construction, consider the paradigmatic case of a single qubit undergoing arbitrary decoherence, whereby $\Omega_{e}^{[\ell]} \equiv \Omega^{(1)}=\operatorname{span}\left\{\sigma_{\alpha} \otimes B_{\alpha}\right\}$, and $\sigma_{\alpha}=\{I, X, Y, Z\}$ are the identity and the Pauli matrices. The corresponding DD group $\mathcal{G}=\mathbb{Z}_{2} \times \mathbb{Z}_{2}$ is (projectively) represented as $\{I, X, Y, Z\}$, and is generated by, e.g., $\Gamma=\{X, Y\}$. EDD is given by $X Y X Y Y X Y X$ [16]. Starting with a $Q^{[\ell]}\left[\tau_{\ell}\right]$ gate, we have:

$$
Q^{[\ell+1]}=Q_{*}^{[\ell]} X^{[\ell]} Y^{[\ell]} X^{[\ell]} Y^{[\ell]} Y^{[\ell]} I_{Q}^{[\ell]} X^{[\ell]} I_{Q}^{[\ell]} Y^{[\ell]} I_{Q}^{[\ell]} X^{[\ell]},
$$

in which $I_{Q}^{[\ell]}$ and $Q_{*}^{[\ell]}$ are defined in Eq. (3), and are themselves combinations of stretched $Q^{[\ell]}$ and $Q^{-1,[\ell]}$. The length of the combined new sequence is given by $\tau_{\ell+1}=\left[14+3 \times 2^{1 /(\ell+1)}\right] \tau_{\ell} \leq 20 \tau_{\ell} \equiv \chi \tau_{\ell}$. Representative simulation results are presented in Fig. 1. Parameters have been chosen to mimic a high-quality silicon (Si) quantum dot, where the electron spin qubit undergoes hyperfine-induced decoherence due to a fraction $\approx 1$ ppm of non-zero spin ${ }^{29}$ Si nuclei (about one order of magnitude larger than currently achieved isotopically purified 


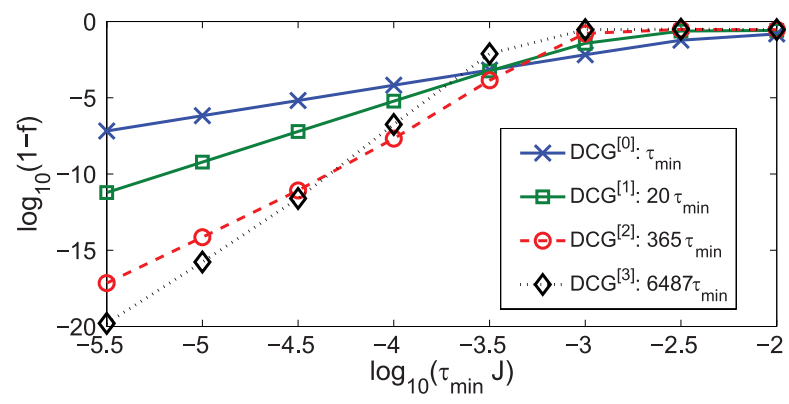

FIG. 1: Fidelity of increasing DCG orders for $Q=$ $\exp [-i(\pi / 3) X]$ applied to $|\psi\rangle=(|0\rangle+|1\rangle) / \sqrt{2}$, and $\rho_{B}$ fully mixed. The fidelity is $f \equiv \operatorname{Tr} \sqrt{\sqrt{\rho_{a}} \rho_{t} \sqrt{\rho_{a}}}$, where $\rho_{t}\left(\rho_{a}\right)$ is the target (actual) final qubit state, and satisfies $1-\Delta \leq f \leq$ $\sqrt{1-\Delta^{2}}$ [28]. The bath spins couple to the central system spin $\mathbf{S}$ via a Heisenberg interaction $H_{S B}=\sum_{i=1}^{5} j_{i} \mathbf{S} \cdot \mathbf{I}^{(i)}$, with $j_{i}$ randomly picked in $[0, J], J \equiv 10$ (arbitrary units). The bath spins $I^{(j)}$ evolve under a dipolar interaction $H_{B}=$ $\sum_{i, j} b_{i j}\left(I_{X}^{(i)} I_{X}^{(j)}+I_{Y}^{(i)} I_{Y}^{(j)}-2 I_{Z}^{(i)} I_{Z}^{(j)}\right)$, with $b_{i j}$ randomly picked in $\left[0,10^{-2}\right]$. Primitive gates were implemented using rectangular pulse shapes to allow for numerically exact simulations. For fixed $J$, reducing $\tau_{\text {min }}$ may be understood in terms of a finer temporal resolution of the resulting 'digitized pulse profile'. Note that at $\log _{10}\left(\tau_{\min } J\right)=-5.5$ the fidelity for $\mathrm{DCG}^{[3]}$ is 13 orders of magnitude better than for $\mathrm{DCG}^{[0]}$, in spite of the gate taking nearly 6500 times longer.

Si [26]). Consistent with the perturbative nature of the error cancellation in dynamical QEC, the improvement due to the recursive design is manifested in the increasing slopes associated with higher concatenation levels once the gating time $\tau_{\min }$ is sufficiently short. Concatenated DCGs may also prove instrumental in reducing gating errors on a recently proposed logical qubit encoded in the singlet/triplet spin manifold of a Si double quantum dot [27], in particular to protect exchange-based logical $Z$ rotations against magnetic field noise. Finally, we expect the same control sequences to be effective for nonMarkovian decoherence induced by unbounded environments with a sufficiently 'hard' spectral cutoff [9].

Conclusion. -We have presented a general constructive solution to the problem of generating arbitrarily accurate quantum gates with finite control resources in an open-loop setting. In addition to settling a fundamental question, our results point to several venues for further investigation. On the theory side, improved constructions should incorporate more realistic local baths and combinatorial Eulerian design [29]. From an implementation perspective, making contact with optimal-control formulations [30] may allow to boost efficiency in experimentally available control platforms.

K. K. and L. V. gratefully acknowledge insightful discussions with Winton Brown. This material is based upon work supported by the NSF under Grants No. PHY-0555417 and PHY-0903727 (to LV), and CCF726439 and PHY-803304 (to DAL). DAL thanks IQICaltech, where part of his work was done.
[1] See, e.g., H. Mabuchi and N. Khaneja, Int. J. Robust Nonlinear Control 15, 647 (2005) for a recent survey.

[2] V. Giovannetti, S. Lloyd, and L. Maccone, Phys. Rev. Lett. 96, 010401 (2006); S. Boixo et al., ibid. 98, 090401 (2007).

[3] M. A. Nielsen and I. L. Chuang, Quantum Computation and Quantum Information (Cambridge University Press, Cambridge, UK, 2000).

[4] E. Knill, Nature 434, 39 (2005).

[5] P. Aliferis, D. Gottesman, and J. Preskill, Quantum Inf. Comput. 6, 97 (2006).

[6] U. Haeberlen, High Resolution NMR in Solids: Selective Averaging (Academic Press, New York, 1976).

[7] L. Viola and S. Lloyd, Phys. Rev. A 582733 (1998); L. Viola, E. Knill, and S. Lloyd, Phys. Rev. Lett. 82, 2417 (1999); L. Viola, S. Lloyd, and E. Knill, ibid. 83, 4888 (1999); L. Viola and E. Knill, ibid. 94, 060502 (2005).

[8] K. Khodjasteh and D. A. Lidar, Phys. Rev. Lett. 95, 180501 (2005); Phys. Rev. A 75, 062310 (2007).

[9] G. S. Uhrig, Phys. Rev. Lett. 98, 100504 (2007); W. Yang and R.-B. Liu, ibid. 101, 180403 (2008).

[10] J. J. L. Morton et al., Nature 455, 1085 (2008).

[11] S. Damodarakurup et al., Phys. Rev. Lett. 103, 040502 (2009).

[12] M. J. Biercuk et al., Nature 458, 996 (2009).

[13] Y. Sagi, I. Almog, and N. Davidson, arXiv: 0905.0286.

[14] K. Khodjasteh and L. Viola, Phys. Rev. Lett. 102, 080501 (2009); Phys. Rev. A 80, 032314 (2009).

[15] J. R. West, B. H. Fong, and D. A. Lidar, arXiv:0908.4490.

[16] L. Viola and E. Knill, Phys. Rev. Lett. 90, 037901 (2003).

[17] G. S. Uhrig and S. Pasini, arXiv:0906.3605.

[18] S. Pasini et al., Phys. Rev. A 80, 022328 (2009).

[19] K. R. Brown, A. W. Harrow, and I. L. Chuang, Phys. Rev. A 70, 052318 (2004).

$[20]\|X\|_{1} \equiv \operatorname{Tr} \sqrt{X^{\dagger} X}$ and $\|X\|$ is the standard operator norm (largest eigenvalue of $\sqrt{X^{\dagger} X}$ ). $\operatorname{Tr}_{X}$ denotes partial trace over the bath $(X=B)$ or system $(S=X)$.

[21] K. Khodjasteh, D. A. Lidar, and L. Viola, in preparation.

[22] Formally, Eq. (2) is a truncation of the Magnus expansion and is valid as long as $\sum_{i}\left\|\bmod _{B} E_{Q_{i}\left[\tau_{i}\right]}\right\|<\pi$, see $[6,14]$.

[23] Note that similarly to $H_{e}, E_{Q^{[\ell]}}$ may be expanded as $E_{Q^{[\ell]}}=\sum S_{\alpha} \otimes B_{Q, \alpha}$, where $B_{Q, \alpha}$ are operators acting on $\mathcal{H}_{B}$ that depend on both $\left\{B_{\alpha}\right\}$ and the control sequence.

[24] If $D=\operatorname{dim}\left(\mathcal{H}_{S}\right)$, in the worst case $\mathcal{G}^{[\ell]}$ may be identified with the 'adversarial DD group' that achieves maximal decoupling on $S, \mathcal{G}_{\text {adv }} \simeq \mathbb{Z}_{D} \times \mathbb{Z}_{D}$, under an irreducible unitary representation in $\mathcal{H}_{S}$. Thus, $d_{\ell} \leq D^{2}$ and $m_{\ell}=2$, for all $\ell[7,29]$. For $n$ qubits, the adversarial error model $\Omega_{e}^{(n)}$ is spanned by all combinations of up to $n$ Pauli matrices, and $\mathcal{G}_{\mathrm{adv}} \simeq\left(\mathbb{Z}_{2} \times \mathbb{Z}_{2}\right)^{\times n}$. Fixing $\Omega_{e}^{[\ell]}=\Omega_{e}^{(n)}$ yields worst-case CDCGs of length exponential in $n$.

[25] H. K. Ng, D. A. Lidar, and J. Preskill, arXiv:0911.3202

[26] N. Tsubouchi et al., Jpn. J. Appl. Phys. 40, L1283 (2001).

[27] J. E. Levy et al., arXiv:0904.0003.

[28] C. Fuchs and J. van de Graaf, IEEE Trans. Inf. Theory 45, 1216 (1999).

[29] P. Wocjan, Phys. Rev. A 73, 062317 (2006).

[30] T. Schulte-Herbrueggen et al., arXiv:quant-ph/0609037; J. Clausen, G. Bensky, and G. Kurizki, Phys. Rev. Lett. 
104, 040401 (2010). 